Journal of Computer Science 8 (7): 1150-1155, 2012

ISSN 1549-3636

(C) 2012 Science Publications

\title{
Learning Software Component Model for Online Tutoring
}

\author{
${ }^{1} \mathrm{M}$. Balakrishnan and ${ }^{2} \mathrm{~K}$. Duraiswamy \\ ${ }^{1}$ Department of Computer Science and Engineering, \\ Selvam College of Technology, Namakkal, Tamilnadu, India \\ ${ }^{2}$ Dean (Academic), K.S. Rangasamy College of Technology, \\ Tiruchengode, Tamilnadu, India
}

\begin{abstract}
Problem statement: Web services are interface elements which allow applications to render functional services to requesting clients using open standard protocols. A lecture method combines both social association and urban processing as course design and delivery is termed as Interface Learning. Many Interface learning services is presenting through online. To make an online tutoring scheme more effective, the previous study used web services and application programs like instant messaging based on environments in which students reside. But the downside is that it is difficult to maintain the service request queues online. The services and data storage processes are inefficient. Approach: To overcome all the above issues, a Learning Software Component Model (LSCM) framework is formed in the present study to build a component model based on communication services available on the network. In addition to this, the proposed software component modeled with Learning Object (LO) aspects integrates the related sub hierarchical components with the main component object framework. Based on LSCM, training schedules are identified efficiently. Results: The proposed LSCM framework is experimented to show the performance improvement with the previous online tutoring scheme based on web services in terms of delivery report, maintenance of tutoring sessions and reliability. Conclusion: Compared to an existing online tutoring through web services, the proposed LSCM framework performance is $75 \%$ better in providing learning services to the providers.
\end{abstract}

Key words: Web services, interactive learning, LSCM, learning object

\section{INTRODUCTION}

Through quick progresses in dispersed multimedia expertise, the concept of synchronized interface distance education has established further consideration than any other interface learning method. While conventional behaviors are distinguished by the intrinsic environmental constraint, the initiations of outlying E-learning requests have facilitated scholars to listen course wherever through an online computer. With existing profitable makes of standard applications, two chief kinds are gladly valid for distant E-learning. To achieve the definite learning intention, a learning service provider is provided by attaining a knowledge comprising of communication infrastructure, conference, educators, instructive objects.

An emerging topic in software engineering is referred to as the Component-Based Development (CBD). The advantage of CBD technology discussed in (Lau and Wang, 2007) is its primary software section representative, which describes the components and their symphony devices. In this study, the author review and examine present component models and categorize them into a classification based on generally established desired data for CBD.

In spite of the materialization of the new creation of Web based education systems, customary education form, in which instructor and learner are interacting with each other in an unchanged classroom, persists to include its unequaled merits. Not like several Web-based education systems embracing in a corresponding way, the instructor brings out education contented online and learners acquire fixed education resources at diverse period, genuine education takes a coordinated education course, which students pay attention to the reside lessons whereas the instructor provides the speech. In the majority situations, coordinated education capture applicants' thought and attention a lot new efficiently than uncomfortable one. As a result, concurrent interface effective education with practice is of requisite consequence in expanse education. The architecture of an E-learning system is described in Fig. 1.

Corresponding Author: Balakrishnan, M., Department of Computer Science and Engineering, Selvam College of Technology, Namakkal, Tamilnadu, India 


\section{J. Computer Sci., 8 (7): 1150-1155, 2012}

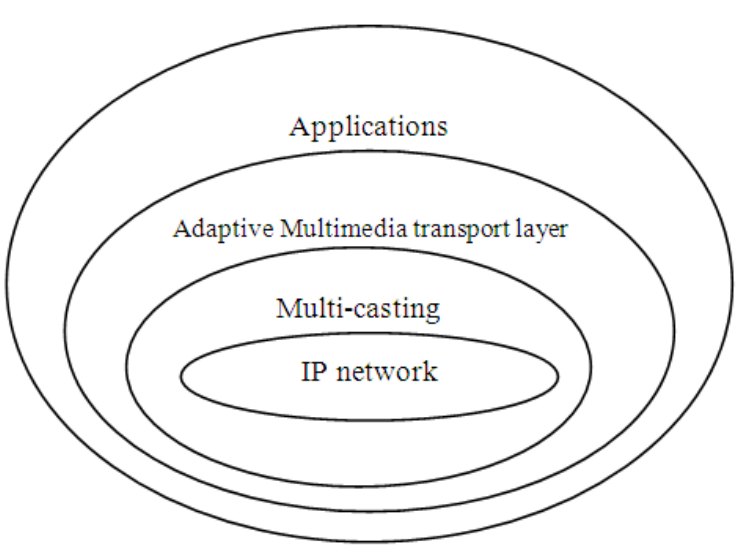

Fig. 1: Architecture diagram of E-learning system

There are numerous issues arising in the proposal of a distant online education system. The bandwidth conditions are low, in efficient real time interactive data. But, classroom-based online education systems assist both the instructor and learners in the educating process and have accomplished flourishing results. Even all web based learning services are provided interactive learning services more efficient, the main drawback is that if number of service requests queued up, then is difficult to sustain and respond all those services for the students. To make an online tutoring scheme more efficient, middleware interactive components are built. A software layer that locates between the system and the function is termed as middleware to provide reusable solutions to regularly met problems like interoperability, security, heterogeneity.

The main contribution of the present study is to build a component software framework which is described in section 3 briefly based on web services and learning object chosen by the students in an appropriate environment.

Literature review: The progression of humancomputing interface is integrated to enhance the work efficiency (Suo et al., 2009). In the education part, human-computing interaction can assist the instructor to lecture the course and assist the learners learn and converse with others. A number of schemes, such as (Allert et al., 2002) and realize the particular humanevaluating communications in their knowledge situation. At the similar time, portable devices such as mobile phone, smart phone, have been straightforwardly available for common motives. Researchers in (Maedche and Staab, 2003) lay emphasis on those mobile devices cooperate a significant function in education. An instance, the instructor used his portable device to fetch the management folder and to organize the folders to visit, whereas the learners can utilize a portable device to converse with others. Several of these mechanisms have been included in several assignments.

A prior elegant Platform facilitates portable devices wandering with customers to join into elegant Space by eADK-based negotiator of elegant Platform. Nevertheless, it is easy for the customers, particularly in Smart Space to utilize their portable devices. Open Smart Platform is used for Web-based mobile edges of Smart Space. It rendered the portable interactions, such as PPT upload, as a mesh page (Doherty and Atkinson, 2004).

Processing of web services using B2B and B2C are essential for reliable transaction processing. Traditional web services relax the property and completely depend on mechanisms to ensure the transaction validity in the presence of failures. In this study (Alrifai et al., 2009) the author proposes an architecture for concurrency control.

Since nearly all the portable devices such as laptop, smart phone, or even ordinary cell phones provided by an incorporated Web browser, it is suitable for the customers to enhance the services and communications inside Smart Space (Friesen et al., 2005). In addition to this, providing reliability and quick delivery for the portable devices to interrelate with Smart Space is enhanced by this mechanism. As a centralized server among the portable devices and components inside Smart Space, when the similar portable device access enhances, OSPG might occupy reliability (Sintek and Decker, 2002) and cache method to improve the whole consignment for Smart Space and too might manage the parallel portable devices to evade the burden of the entire education system.

Event monitoring is essential to Web service requirements. The study (Wang et al., 2009) introduces a real time scrutinizing scheme for Web service necessities. It comprises a measurement of service constrictions that links to examine necessities and additionally includes a supervising form that envelops five types of scheme actions applicable to customer request, response, submission, source and organization and a supervising structure in which diverse probes and agents gather actions and data that are receptive to necessities.

In machine learning and retrieval of information the use of positive an unlabeled data is very essential. Several methodologies for resolving this dilemma have been planned but they do not function properly whilst only a diminutive data are accessible. Zhou et al. (2010) the author presented a new technique called Topic Sensitive PLSA to resolve the diminutive data problem. This technique uses a less information from the customer. The regulation of information is stored as a set of constraints. The author developed a repetitive algorithm that can attain the confined optimal of the objective function. 
To improve the online tutoring scheme more effective, we implement learning software component model for communication services like messaging, chatting. The learning software component is modeled with learning object aspects to build a component model.

\section{MATERIALS AND METHODS}

The proposed Learning Software Component Model is designed to build a framework for online tutoring scheme which run under web services. It renders the communication services to applications like messaging, chatting. The proposed LSCM architecture made up of two strategies. The initial strategy is to build a component model framework based on web related communication services. The second strategy is to provide the communication services through the LSC framework to the students participated in online tutoring schemes. The architecture diagram for the proposed LSCM framework is shown in Fig. 2.

In the learning software component building strategy, the learning communication services are analyzed based on students' environment. The learning software component framework is built with learning services and each component is built with the learning objects chosen by the students. Each component contains an appropriate service based on respective students' level functionality.

The second strategy is in the process of communication services through the component invocation based on LO on the student required levels. Before delivering the service of the component to the student, the authorization of the student is interpreted based on their skills. So, only the authorized and demanded communication services are delivered to the respective environmental application student.

Using learning software components of services, the framework is built which has several types of communication services like messaging, chatting etc. Based on the interactive component based middleware service architecture, the components are built with the services and it efficiently achieving the interoperability service by providing a learning service to the students. The proposed component based middleware service architecture consumes less time for building the components and less storage services.

Procedure for learning software component building phase: The LSCM is built based on the learning services provided by the online tutoring scheme. Based on communication services like messaging, chatting and the component are built with an appropriate service and LSC model. The process of LSCM is shown in Fig. 3. The procedure describes the LSCM process is:

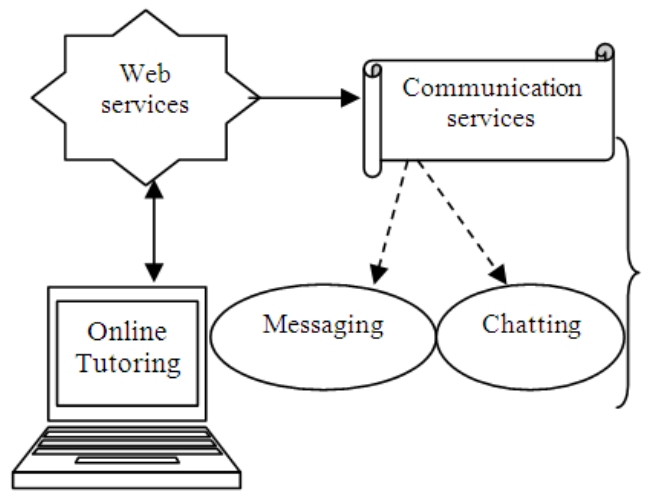

Based on services

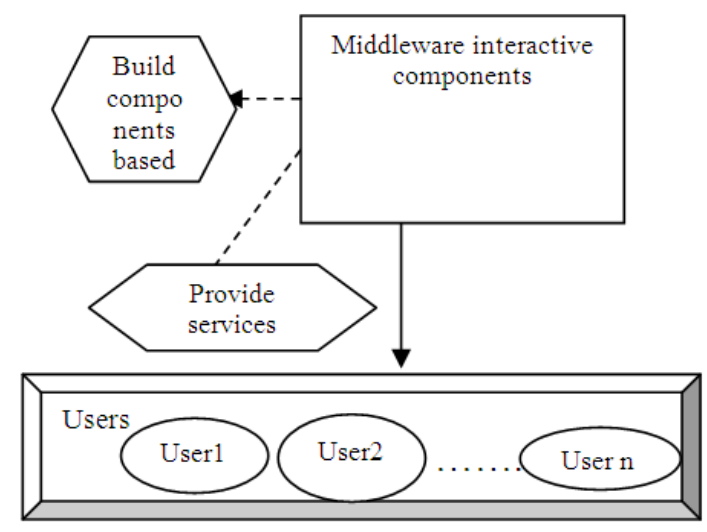

Fig. 2: Architecture diagram for LSCM for online tutoring scheme through web services

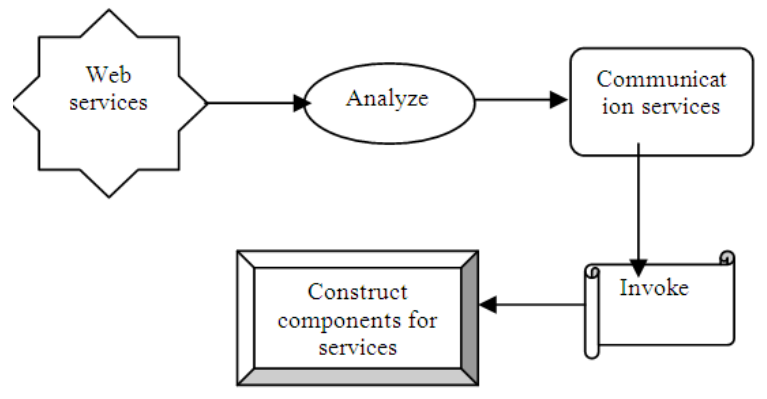

Fig. 3: Process of LSCM framework

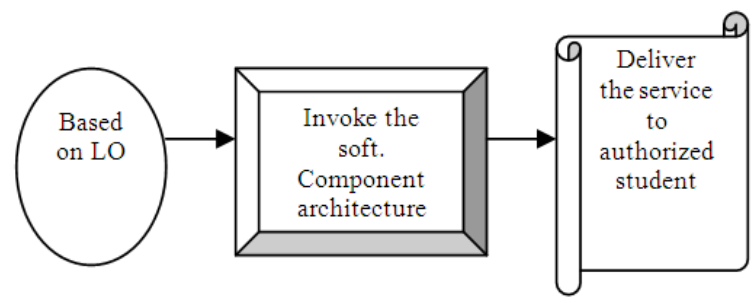

Fig. 4: Steps for communication services 


\section{J. Computer Sci., 8 (7): 1150-1155, 2012}

Step 1: Input: Different types of web services

Step 2: Analyze the type of services

Step 2.1: Communication services: Messaging, chatting

Step 3: Based on students' request for service,

Step 3.1: Invoke the services

Step 4: Based on services

Step 4.1: Build structural components

Step 4.2: Construct the set of components

Step 4.3: Invoke the services for different set of components

Step 4.4: Each component may have a different set of services

Step 5: Output: Build the components efficiently.

The process of building the component framework based on communication services is described in Fig. 3. The applications may have several services interact with the other services. Based upon the communication services, the component is built using the learning software Component Based Middleware service architecture with corresponding components based communication services.

Procedure to provide communication service through LSCM framework: The procedures below described the process of providing communication service (Fig. 4) through LSCM framework:

Step 1: Based on Learning Object (LO),

Step 1.1: Invoke the software component architecture from LSCM

Step 2: Before delivering the service,

Step 2.1: Identify the authorization of the student, based on their skills

Step 3: Deliver the demanded service component to the student.

Based on communication service component, the component function is evaluated to show the coordinate value of the message event. Render the services to students' applications need. After rendering the services, only the authorized students can demand the services are applicable to the student environment.

\section{RESULTS}

The Learning Software Component Model (LSCM) is implemented by using the Java platform. The researches were scuttled on an Intel P-IV appliance with $4 \mathrm{~GB}$ memory and $3 \mathrm{GHz}$ dual processor CPU. The performance evaluation tests aimed at comparing the online tutoring scheme with challenging interactions through web services. LSCM framework is depended on interception (Fig. 2). At set up it build the components based on communication services rendered. The service used with component building architecture to raise the online tutoring scheme more efficient. A LSCM framework carries two types of operations generic (building components based on LO, service rendering...). Operations can be assigned to different services and components in the infrastructure. The performance of LSCM is evaluated by metrics such as Delivery report rate, Maintenance of tutoring sessions, Reliability.

Delivery Report Rate: Amount of time taken to deliver the service to the students whoever involved in the online tutoring process. Figure 5 describes the delivery report rate for students who are in need of online tutoring. Many users are participating in the experimentation to validate LSCM.

Comparison result of LSCM with an existing online tutoring through web services based on the delivery report rate, measured in terms of milliseconds $(\mathrm{m} / \mathrm{s})$. When the number of students' service requests applications increases, the delivery report rate based on communication services is high in the proposed LSCM contrast to an existing online tutoring scheme through web services. The performance graph of the proposed LSCM in component building phase is shown in the Fig. 5. The variance in the delivery report rate would be $12-20 \%$ high in the proposed LSCM.

Maintenance of tutoring sessions: This metric describes how the tutoring sessions are maintained based on services, tutoring process and the time taken to analyze the skills of the students' and to learn it. Figure 6 describes the maintenance of tutoring sessions even when number of users increases. Since the services are built as a component framework, the maintenance of tutoring sessions was easy.

When the number of students' service requests applications increases, the maintenance of tutoring sessions is also being high in the proposed LSCM contrast to an existing online tutoring scheme through web services. The performance graph of the proposed LSCM in component building phase is shown in the Fig. 6. The variance in the maintenance would be 15$20 \%$ high in the proposed LSCM.

Reliability: The ability of the proposed LSCM framework to achieve and continue its functions in regular circumstances, as well as aggressive or unpredicted circumstances.

Figure 7 describes the reliability of the proposed LSCM with an existing online tutoring scheme through web services. Various numbers of applications are used in the experimentation to improve the reliability of LSCM. Comparison result of LSCM with an existing online tutoring scheme through web services shows that the proposed LSCM perform better. The reliability of the proposed LSCM also be improved. 


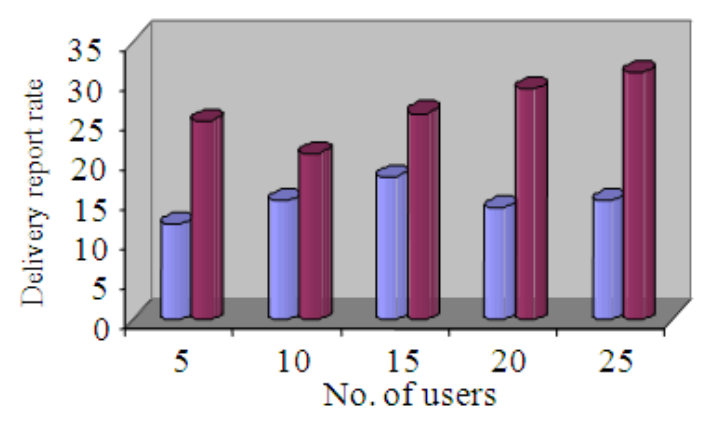

$\square$ Existing online tutoring scheme through web services $\checkmark$ Proposed LSCM for online tutoring scheme

Fig. 5: Users Vs. delivery report rate

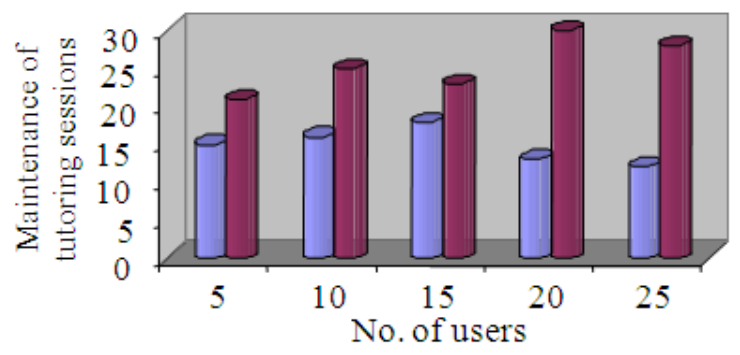

$\square$ Existing online tutoring scheme through web services $\square$ Proposed LSCM for online tutoring scheme

Fig. 6: Users Vs. Maintenance of tutoring sessions

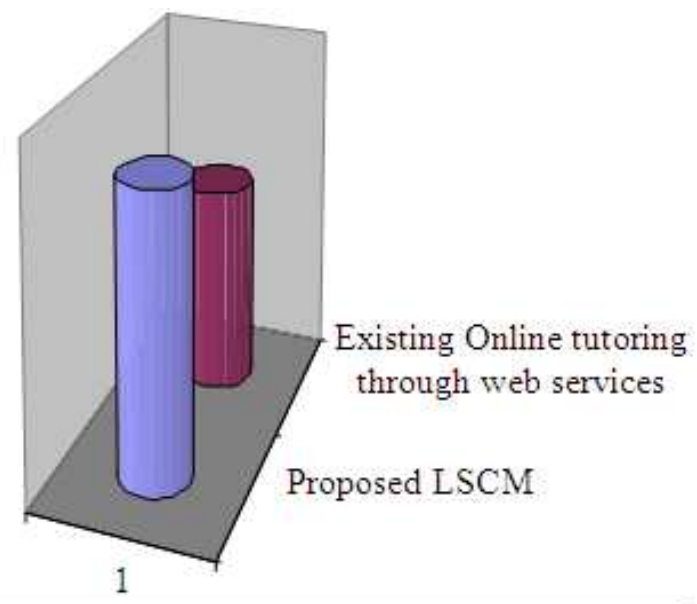

0 Proposed LSCM

Existing Online tutoring through web services

Fig. 7: Reliability model for both SCM and online tutoring through web services

\section{DISCUSSION}

In the proposed study, a component can be designed for communication service composition to identify the general design mechanisms for online tutoring scheme scripting in mainstream languages such as Java. We run independent tests with a growing number of applications and number of students' service requests responded by each trainer is $30-35 \%$ requests per client. Compared to an existing online tutoring schemes through web services, the proposed LSCM framework achieved an effective tutoring services through online by building the component model based on an appropriate service.

Finally, it is observed that the proposed LSCM framework is efficiently built based on learning object and the web communication services are also rendered to users' based on their environment. The reliability and performance rate also be improved in the proposed LSCM framework.

\section{CONCLUSION}

The progression of proposed LSCM for online tutoring has showed us that the growth of a suitable culture for online tutoring is rapidly high. According to the users' need, the learning services are offered to the students by building the component for communication services efficiently. The middleware interactive service components are developed for building the service components efficiently. A desirable feature of the proposed LSCM framework is high maintenance of tutoring session based on communication services. LSCM with the service rendering approach allows trainers to interact with the students based on learning object aspects and communication services. The experimental results showed that the improved performance of delivery time, maintenance rate and reliability.

\section{REFERENCES}

Allert, H., C. Richter and W. Nejdl, 2002. Learning objects on the semantic web-explicitly modelling instructional theories and paradigms. The Pennsylvania State University.

Alrifai, M., P. Dolog, W.T. Balke and W. Nejdl, 2009. L3S Res. Center, Leibniz Univ. of Hannover, Hannover, Germany, Distributed Management of Concurrent Web Service Transactions. IEEE Trans. Serv. Comput. 2: 289-302. DOI: 10.1109/TSC.2009.29

Doherty, B. and M. Atkinson, 2004. A pilot study of online tutoring using Smarthinking. 
Friesen, N., A. Roberts and S. Fisher, 2005. Cancore: Metadata for learning objects. Canadian J. Lear. Technol.

Lau, K.K. and Z. Wang, 2007. Software Component Models. IEEE Trans. Software Eng., 33: 709-724. DOI: 10.1109/TSE.2007.70726

Maedche, A. and S. Staab, 2003. Services on the movetowards p2p-enabled semantic web services. Proceedings of the 10th International Conference on Information Technology and Travel and Tourism, Jan. 29-31, Springer, Helsinki.

Sintek, M. and S. Decker, 2002. Triple-a query, inference and transformation language for the semantic web. The Semantic Web- ISWC, 2342: 364-378. DOI: 10.1007/3-540-48005-6_28
Suo, Y., N. Miyata, H. Morikawa, T. Ishida and Y. Shi, 2009. Open smart classroom: Extensible and scalable learning system in smart space using web service technology. IEEE Trans. Knowl. Data Eng., 21: 814-828. DOI: 10.1109/TKDE.2008.117

Wang, Q., J. Shao, F. Deng, Y. Liu and M. Li et al., 2009. An online monitoring approach for web service requirements. IEEE Trans. Serv. Comput. 2: 338-351. DOI: 10.1109/TSC.2009.22

Zhou, K., X. Gui-Rong, Q. Yang and Y. Yu, 2010. Learning with positive and unlabeled examples using topic-sensitive PLSA. IEEE Trans. Knowl. Data Eng., 22: 46-58. DOI: 10.1109/TKDE.2009.56 